bioRxiv preprint doi: https://doi.org/10.1101/211334; this version posted November 15,2017 . The copyright holder for this preprint (which was not certified by peer review) is the author/funder, who has granted bioRxiv a license to display the preprint in perpetuity. It is made available under aCC-BY 4.0 International license.

Phylogenetics

\title{
The Bio::Phylo libraries for phylogenetic data analysis, version 2.0
}

\author{
Rutger A. Vos ${ }^{1,2 *}$ and Hannes Hettling ${ }^{1}$ \\ ${ }^{1}$ Naturalis Biodiversity Center, Leiden, P.O. Box 9517, 2300RA, The Netherlands \\ ${ }^{2}$ Institute of Biology Leiden, Leiden University, Leiden, P.O. Box 9500, 2300RA, The Netherlands \\ ${ }^{*}$ To whom correspondence should be addressed.
}

\begin{abstract}
Motivation: Phylogenetic analysis is a broad and expanding field that requires versatile programming toolkits to manage the various data types, file formats, and needs for scalability, simulation, visualization, and data exploration.

Results: We present version 2.0 of the Bio::Phylo libraries for phylogenetic data analysis. This new release represents a rewrite of the architecture, allowing for extensions that improve speed and persistence, as well as increased functionality in terms of analysis, data reading and writing, and visualization.

Availability: The package is released as open source software under the same terms as Perl itself and available from the comprehensive Perl archive network as well as directly from the source code repository.

Contact: rutger.vos@naturalis.nl

Supplementary information: Supplementary data are available as do $1: 10.5281 /$ zenodo. 1039210
\end{abstract}

\section{Introduction}

Phylogenetic data is multi-faceted, encompassing morphological observations, molecular sequences, biological taxa, and tree and network topologies, and is ubiquitous in a variety of research fields including comparative genomics, systematics, evolution, biodiversity research, and ecology. The types of operations that are performed on phylogenetic data range from data cleaning, integration, and conversions, to simulation, character analysis and inference, and visualization. Flexible programming toolkits that operate on phylogenetic data and aid in scripting these operations are therefore very useful and available in a variety of programming languages. Bio::Phylo (Vos et al., 2011) is the most versatile toolkit for handling phylogenetic data in the Perl programming language.

Conceived about ten years ago (Vos, 2006, 2017), Bio::Phylo has been under ongoing development ever since. Numerous people have contributed to it, by writing code - as volunteers and Google Summer of Code students - by submitting bug reports, and by providing inspiration and impetus in larger collaborative networks (e.g. Stoltzfus et al. (2010), Koureas et al. (2016b), Koureas et al. (2016a)) and hackathons (e.g. Lapp et al. (2007), Katayama et al. (2010), Katayama et al. (2011), Katayama et al. (2013), Stoltzfus et al. (2013), Katayama et al. (2014), Vos et al. (2014)). In the process, additional requirements surfaced, which have been addressed by a rewrite of the internal architecture and the addition of functional modules. These have been integrated in a version 2.0.0 release, which we present here.

\section{Design}

In 'object-oriented' programming, the different concepts within the problem domain that the software addresses are modelled as different classes (e.g. a class to model phylogenetic trees, and one to model multiple sequence alignments) that inherit some of their functionality from other such classes. For example, a class that represents the blueprint for a multiple sequence alignment might inherit certain generalized attributes of character state matrices (e.g. the number of characters and taxa) from a 'super-class'. In turn, this multiple sequence alignment class can be inherited from to create a blueprint for a more specialized class, for example one that models amino-acid alignments.

The object-oriented programming paradigm allows other programmers to extend the functionality of toolkits by inheriting from classes to address additional use cases, for example by improving performance in various ways. Bio::Phylo has always allowed for this, but the version 1.0 design made this relatively cumbersome because a lot of functionality would have to be re-implemented for any newly created class to integrate well in the rest of the toolkit. In the new design, each class has been reworked into 
bioRxiv preprint doi: https://doi.org/10.1101/211334; this version posted November 15, 2017. The copyright holder for this preprint (which was not certified by peer review) is the author/funder, who has granted bioRxiv a license to display the preprint in perpetuity. It is made available 2 under aCC-BY 4.0 International license.

Table 1. Large, published phylogenies made available as database files

\begin{tabular}{ll}
\hline Project & Database files \\
\hline PhyloTree (van Oven and Kayser, 2009) & 10.6084/m9.figshare.4620757.v1 \\
D-Place (Kirby et al., 2016) & 10.6084/m9.figshare.4620217.v1 \\
NCBI taxonomy (Federhen, 2011) & 10.6084/m9.figshare.4620733.v1 \\
Greengenes (DeSantis et al., 2006) & 10.6084/m9.figshare.4620214.v1
\end{tabular}

two separate modules, one that performs the actual changes to any data associated with the class, and one that performs all the other user-friendly operations that do not directly affect the data. With this new design, a new class that only re-implements the changes to the data can re-use all the other operations, and thus be much more compact and easily written. In addition, the integration of such new classes has been made far more flexible (by adopting, in the parlance of software design patterns (Gamma et al., 1995), the 'Factory' pattern throughout).

The usefulness of this change of design is demonstrated by two optional extension packages that can be installed alongside Bio::Phylo. One of them, Bio::PhyloXS, serves as a drop-in replacement of the core data objects in Bio::Phylo, re-implemented in the $\mathrm{C}$ programming language. Because $\mathrm{C}$ is a very fast, compiled language, having some of the core functionality (e.g. setting and fetching data properties) leads to significant speed increases: a simple benchmark test where a small tree topology is constructed and then traversed executes about $700 \%$ faster using this extension. (Note, however, that bindings between $\mathrm{C}$ and Perl are fraught with challenges due to the complexity of the Perl API and the differences in memory management on different architectures. Hence, this application should currently be considered 'experimental'.) The second optional extension, Bio::Phylo::Forest::DBTree, allows very large trees to be stored into, and accessed from, a simple SQLite database. This means that these trees never have to be loaded in working memory, and that, once stored in the database, there is no "file reading" step. The typical application for this is to store static, immutable trees. To demonstrate this, we make available database files that were created by indexing the releases listed in Table 1.

\section{Data input and output}

In addition to the file formats already supported in previous releases of Bio::Phylo, several new formats are now read and/or written. Specifically, PhyloXML (Han and Zmasek, 2009), New Hampshire eXtended (NHX, Zmasek and Eddy (2001)), the extensions added to the NEXUS standard by the programs TreeAnnotator and Figtree (Rambaut, 2007), and a 'badgerfish' mapping of NeXML (Vos et al., 2012) to JSON can now both be read and written. In addition, several response formats from web services (namely, the DarwinCore archive format returned by GBIF (Baker et al., 2014), the response format from the TaxoSaurus.org service (Stoltzfus et al., 2013), and the response documents of uBio.org) as well as FASTQ (Cock et al., 2009) files can be read. Data files for Hennig86 (Farris, 1988) and for the haplotype 'Network' program (fluxus-engineering.com) can be written.

Support for RDF is experimental: RDF/XML triples can be written by way of a two-step process that first exports data as NeXML and then transforms this to statements that obtain terms from CDAO (Prosdocimi et al., 2009); this is the same approach and uses the same transformation stylesheet as TreeBASE (Piel et al., 2009)). RDF graphs can also be 'read' in the sense that a graph can be loaded and interrogated with SPARQL queries to extract statements that map onto Bio::Phylo's object model. At present, this means that annotated taxa and tree topologies can be extracted from CDAO/RDF, but character data cannot.

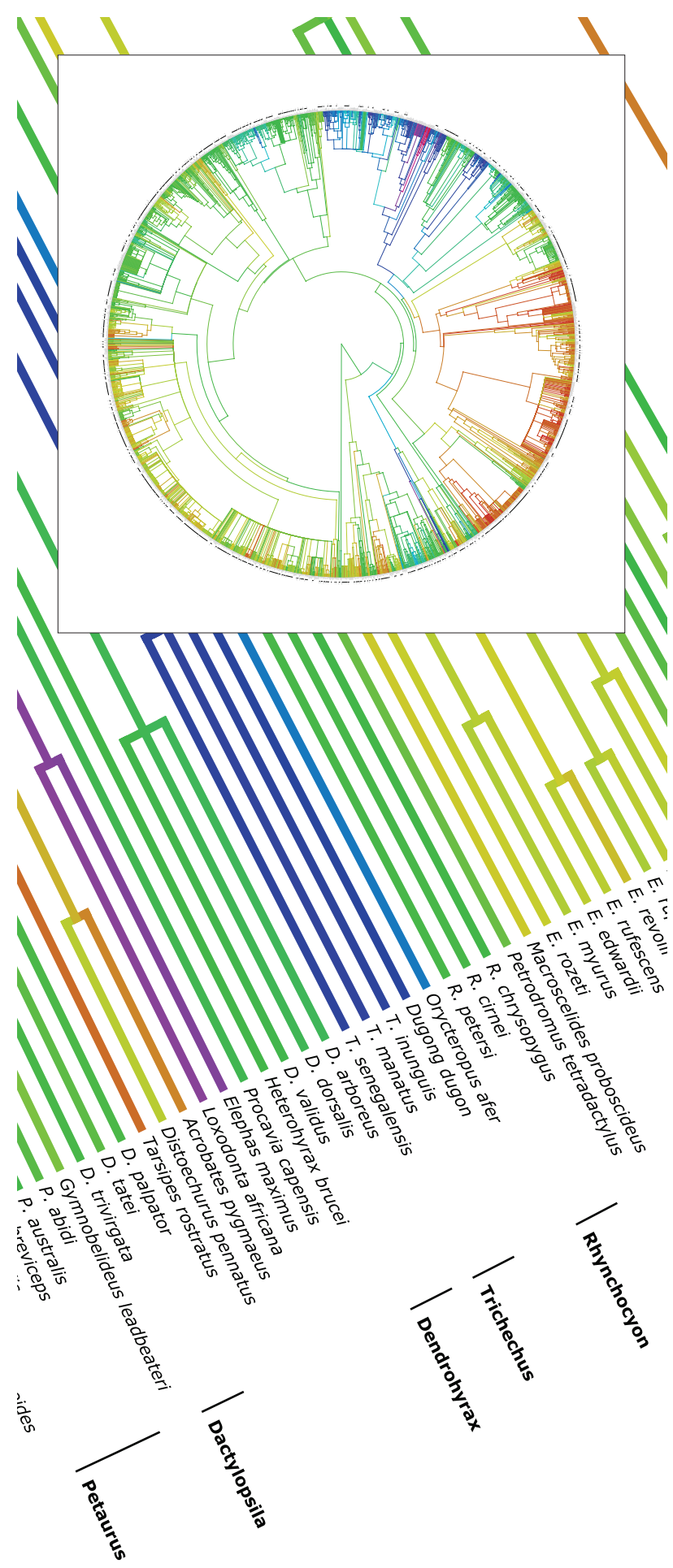

Fig. 1. Mammal supertree (Bininda-Emonds et al., 2007) decorated with log-transformed body mass (Jones et al., 2009) as branch colors and monophyletic genera as labeled braces; detail and full tree (inset). Image produced as per the workflow described on http://rvosa.github.io/bio-phylo/doc/examples/integration/

\section{Analysis, modification, and simulation}

From its first versions onwards, Bio::Phylo has implemented numerous algorithms for computing topological indices, simulating new topologies, and adjusting them algorithmically. Some of the simulation methods were 
developed and discussed by Hartmann et al. (2010), while some of the topology index algorithms were explored by Martyn et al. (2012). To the existing topology indices, some additional methods were added: the Euclidean distance between trees (see Kuhner and Felsenstein (1994)) and the number of 'cherries' in a given topology (see McKenzie and Steel (2000)) can now be computed. To the algorithms that adjust topologies have been added methods to obtain ultrametric trees using the mean path lengths method of Britton et al. (2002), by making node ages proportional to clade size (as per Grafen (1989)), and using an implementation of Stadler's algorithm for computing the relative order of speciation or coalescence events on a given phylogeny (Gernhard et al., 2006).

By implementing a bridge between Bio::Phylo's library code and the $\mathrm{R}$ programming language, additional functionality has become accessible. So far, this has been wrapped in methods to estimate the parameters of the birth/death process and use these to simulate replicates of the input tree (using 'ape', Paradis et al. (2004)); and methods to estimate the parameters of state transition models for binary characters and DNA sequences (using 'phangorn', Schliep (2010); 'phylosim', Sipos et al. (2011); and 'phytools', Revell (2012)). To encapsulate these state transition models, a class hierarchy has been implemented that represents some of the common substitution models (i.e. JC69, HKY85, GTR, F81, K80) such that they can be serialized to the syntax of commonly used programs for phylogenetic inference. In addition, this facility to select substitution models has been combined with a (tree-based) sequence simulator to allow multiple sequence alignments to be replicated.

\section{Visualization}

The ability to visualize phylogenies as rooted, rectangular cladograms and phylograms in vector formats (SVG being best supported) has always existed in Bio::Phylo. This included functionality to paint branches, add pie charts to nodes, collapse clades as triangles, and influence styling (such as branch thickness; fonts and their size, weight, and style). To this has been added in v2.0.0 the ability to draw unrooted and radial tree projections, and the option to mark up higher taxa in labeled braces (straight or arched, depending on projection). Figure 1 demonstrates some of this new functionality.

\section{Impact and re-use}

As of time of writing (October 2017), the first version of Bio::Phylo has been cited 35 times. Among these, the papers with the highest impact used Bio::Phylo in analyses in phylogenomics and comparative genomics (e.g. see Roure et al. (2012); Hayward et al. (2013); De Smet et al. (2013)).

Several infrastructural projects depend on Bio::Phylo. These include the BioPerl project (Stajich et al., 2002), which uses it for reading and writing NeXML. This usage is promoted by the interface compatibility between the two projects: the core data objects of Bio::Phylo, i.e. trees, tree nodes, sequence alignments, and so on, can be used directly in BioPerl. Also, the TreeBASE project (Piel et al., 2009) uses Bio::Phylo for certain server-side maintenance tasks, and the SUPERSMART project (Antonelli et al., 2017), as well as the BioVeL services that expose it (Hardisty et al., 2016), are deeply integrated with Bio::Phylo (and drove some of the development of new functionality).

In addition, several single-purpose programs and pipelines use Bio::Phylo. These include the Monophylizer web service for assessing monophyly in gene trees (Mutanen et al., 2016; Pentinsaari et al., 2016); the CopyRighter tool for improving the accuracy of microbial community profiles through lineage-specific gene copy number correction (Angly et al., 2014); and the PhyloMatch pipeline to discover highly phylogenetically informative genes in sequenced genomes (Ramazzotti et al., 2012).

\section{Availability}

All revisions of the source code are available from the source code repository at http://github.com/rvosa/bio-phylo. The latest stable release version, which over time may fall behind the latest source code revision, is available from the Comprehensive Perl Archive Network (CPAN) at http://search.cpan.org/dist/Bio-Phylo. Accompanying this publication is a uniquely identifiable release, stamped with a Digital Object Identifier (DOI) issued by Zenodo.org: doi:10.5281/zenodo. 1039210.

As is a common convention in Perl software releases, a dual licensing scheme applies to Bio::Phylo - both the Artistic License (https://github.com/rvosa/bio-phylo/blob/master/COPYING) as well as the GNU General Public License (https://github.com/rvosa/biophylo/blob/master/LICENSE) applies. This is generally interpreted to mean that you are free to choose whichever of these licenses fits best with your own project, should you want to reuse all (or part) of Bio::Phylo. This is certainly the spirit: feel free to use these libraries however you see fit. No warranties.

\section{Acknowledgements}

The following people have contributed code to the project: Florent Angly, Jason Caravas, Klaas Hartmann, Mark A. Jensen, Moritz Lenz, Chase Miller, Aki Mimoto, and Jan Willem Wijnands. The following people have provided feedback through bug reports and reviews: Denis Baurain, Chris Fields, Shlomi Fish, Jean-Marc Frigerio, Andreas J. König, Hilmar Lapp, Nicolas Lenfant, Sébastien Moretti, Slaven Rezić, Seiler, and scorpio17. Mannis van Oven has been very helpful in providing the data dumps of the PhyloTree.org project that were indexed as database files (in Table 1). The principal investigators of the labs in which RAV has done his research have all allowed and encouraged the development of Bio::Phylo. These are Arne Mooers, Wayne Maddison, and Mark Pagel, and now Naturalis Biodiversity Center. We are grateful to all these people for their support.

\section{Funding}

The research leading to these results has received funding from the European Community's Seventh Framework Programme (FP7/20072013) under grant agreement no. 237046.

\section{References}

Angly, F. E. et al. (2014). CopyRighter: a rapid tool for improving the accuracy of microbial community profiles through lineage-specific gene copy number correction. Microbiome, 2(1), 11.

Antonelli, A. et al. (2017). Toward a self-updating platform for estimating rates of speciation and migration, ages, and relationships of taxa. Systematic Biology, 66(2), 152-166.

Baker, M. E., Rycroft, S., and Smith, V. S. (2014). Linking multiple biodiversity informatics platforms with Darwin Core Archives. Biodiversity data journal, (2). Bininda-Emonds, O. R. et al. (2007). The delayed rise of present-day mammals. Nature, 446(7135), 507-512.

Britton, T. et al. (2002). Phylogenetic dating with confidence intervals using mean path lengths. Molecular phylogenetics and evolution, 24(1), 58-65.

Cock, P. J., Fields, C. J., Goto, N., Heuer, M. L., and Rice, P. M. (2009). The sanger fastq file format for sequences with quality scores, and the solexa/illumina fastq variants. Nucleic acids research, 38(6), 1767-1771. 
De Smet, R. et al. (2013). Convergent gene loss following gene and genome duplications creates single-copy families in flowering plants. Proceedings of the National Academy of Sciences, 110(8), 2898-2903.

DeSantis, T. Z. et al. (2006). Greengenes, a chimera-checked 16S rRNA gene database and workbench compatible with ARB. Applied and environmental microbiology, 72(7), 5069-5072.

Farris, J. (1988). Hennig86, version 1.5. Distributed by the author, Port Jefferson Station, $N Y$.

Federhen, S. (2011). The NCBI taxonomy database. Nucleic acids research, 40(D1), D136-D143.

Gamma, E. et al. (1995). Design patterns: elements of reusable object-oriented software. Addison-Wesley.

Gernhard, T. et al. (2006). Estimating the relative order of speciation or coalescence events on a given phylogeny. Evolutionary Bioinformatics Online, 2, 285.

Grafen, A. (1989). The phylogenetic regression. Philosophical Transactions of the Royal Society of London. Series B, Biological Sciences, 326(1233), 119-157.

Han, M. V. and Zmasek, C. M. (2009). phyloXML: XML for evolutionary biology and comparative genomics. BMC bioinformatics, 10(1), 356.

Hardisty, A. R. et al. (2016). BioVeL: A virtual laboratory for data analysis and modelling in biodiversity science and ecology. BMC ecology, 16(1), 49.

Hartmann, K. et al. (2010). Sampling trees from evolutionary models. Systematic Biology, 59(4), 465-476.

Hayward, A. et al. (2013). Broad-scale phylogenomics provides insights into retrovirus-host evolution. Proceedings of the National Academy of Sciences, 110(50), 20146-20151.

Jones, K. E. et al. (2009). PanTHERIA: a species-level database of life history, ecology, and geography of extant and recently extinct mammals. Ecology, 90(9), 2648-2648.

Katayama, T. et al. (2010). The DBCLS BioHackathon: Standardization and interoperability for bioinformatics web services and workflows. Journal of biomedical semantics, $\mathbf{1}, 8$.

Katayama, T. et al. (2011). The 2nd DBCLS BioHackathon: Interoperable bioinformatics web services for integrated applications. Journal of biomedical semantics, 2(1), 4.

Katayama, T. et al. (2013). The 3rd DBCLS BioHackathon: improving life science data integration with semantic web technologies. Journal of Biomedical Semantics, 4(1), 6 .

Katayama, T. et al. (2014). BioHackathon series in 2011 and 2012: penetration of ontology and linked data in life science domains. Journal of biomedical semantics, 5,5

Kirby, K. R. et al. (2016). D-PLACE: A global database of cultural, linguistic and environmental diversity. PLOS ONE, 11(7), e0158391.

Koureas, D. et al. (2016a). Community engagement: The 'last mile' challenge for european research e-infrastructures. Research Ideas and Outcomes, 2, e9933.

Koureas, D. et al. (2016b). Unifying european biodiversity informatics (BioUnify). Research Ideas and Outcomes, 2, e7787.

Kuhner, M. K. and Felsenstein, J. (1994). A simulation comparison of phylogeny algorithms under equal and unequal evolutionary rates. Molecular biology and evolution, 11(3), 459-468.

Lapp, H. et al. (2007). The 2006 NESCent phyloinformatics hackathon: A field report. Evolutionary Bioinformatics Online, 3, 287.
Martyn, I. et al. (2012). Computing evolutionary distinctiveness indices in large scale analysis. Algorithms for Molecular Biology, 7(1), 6.

McKenzie, A. and Steel, M. (2000). Distributions of cherries for two models of trees. Mathematical biosciences, 164(1), 81-92.

Mutanen, M. et al. (2016). Species-level para- and polyphyly in DNA barcode gene trees: Strong operational bias in European Lepidoptera. Systematic Biology, 65(6), 1024-1040.

Paradis, E. et al. (2004). APE: analyses of phylogenetics and evolution in r language. Bioinformatics, 20(2), 289-290.

Pentinsaari, M. et al. (2016). Algorithmic single-locus species delimitation: effects of sampling effort, variation and non-monophyly in four methods and 1870 species of beetles. Molecular Ecology Resources, 17(3), 393âŁ"404.

Piel, W. H. et al. (2009). TreeBASE v. 2: A database of phylogenetic knowledge. In e-BioSphere 2009

Prosdocimi, F. et al. (2009). Initial implementation of a comparative data analysis ontology. Evolutionary Bioinformatics, 5, 47.

Ramazzotti, M. et al. (2012). A computational pipeline to discover highly phylogenetically informative genes in sequenced genomes: application to Saccharomyces cerevisiae natural strains. Nucleic Acids Research, 40(9), 38343848.

Rambaut, A. (2007). FigTree, a graphical viewer of phylogenetic trees. See http://tree.bio.ed.ac.uk/software/figtree.

Revell, L. J. (2012). phytools: an R package for phylogenetic comparative biology (and other things). Methods in Ecology and Evolution, 3(2), 217-223.

Roure, B., Baurain, D., and Philippe, H. (2012). Impact of missing data on phylogenies inferred from empirical phylogenomic data sets. Molecular biology and evolution, 30(1), 197-214

Schliep, K. P. (2010). phangorn: phylogenetic analysis in R. Bioinformatics, page btq706.

Sipos, B. et al. (2011). PhyloSim-Monte Carlo simulation of sequence evolution in the R statistical computing environment. BMC bioinformatics, 12(1), 104

Stajich, J. E. et al. (2002). The Bioperl toolkit: Perl modules for the life sciences. Genome research, 12(10), 1611-1618.

Stoltzfus, A. et al. (2010). EvolO: Community-driven standards for sustainable interoperability. Available from Nature Precedings.

Stoltzfus, A. et al. (2013). Phylotastic! making tree-of-life knowledge accessible, reusable and convenient. BMC bioinformatics, 14(1), 158.

van Oven, M. and Kayser, M. (2009). Updated comprehensive phylogenetic tree of global human mitochondrial DNA variation. Human Mutation, 30(2), E386-E394.

Vos, R. A. (2006). Inferring large phylogenies: The big tree problem. Ph.D. thesis, Simon Fraser University.

Vos, R. A. (2017). Design patterns in phylogenetics: Practical tree data structures and objects for serialization. https://doi.org/10.6084/m9.figshare.4524569.v1.

Vos, R. A. et al. (2011). Bio::Phylo - phyloinformatic analysis using perl. BMC Bioinformatics, 12(1), 63.

Vos, R. A. et al. (2012). NeXML: Rich, extensible, and verifiable representation of comparative data and metadata. Systematic biology, 61(4), 675-689.

Vos, R. A. et al. (2014). Enriched biodiversity data as a resource and service. Biodiversity data journal, (2).

Zmasek, C. M. and Eddy, S. R. (2001). ATV: Display and manipulation of annotated phylogenetic trees. Bioinformatics, 17(4), 383-384. 